\title{
HARPS spectropolarimetry of Herbig Ae/Be stars ${ }^{\star}$
}

\author{
S. Hubrig ${ }^{1, \star \star}$, I. Ilyin ${ }^{1}$, M. Schöller ${ }^{2}$, and G. Lo Curto ${ }^{2}$ \\ 1 Leibniz-Institut für Astrophysik Potsdam (AIP), An der Sternwarte 16, 14482 Potsdam, Germany \\ 2 European Southern Observatory, Karl-Schwarzschild-Str. 2, 85748 Garching bei München, Germany
}

Received date / Accepted date

Key words techniques: polarimetric — stars: pre-main sequence — stars: atmospheres — stars: magnetic field — stars: variables: general stars: variables: Herbig Ae/Be

\begin{abstract}
Our knowledge of the presence and the strength of magnetic fields in intermediate-mass pre-main-sequence stars remains very poor. We present new magnetic field measurements in six Herbig Ae/Be stars observed with HARPS in spectropolarimetric mode. We downloaded from the European Southern Observatory (ESO) archive the publically available HARPS spectra for six Herbig Ae/Be stars. Wavelength shifts between right- and left-hand side circularly polarised spectra were interpreted in terms of a longitudinal magnetic field $\left\langle B_{\mathrm{z}}\right\rangle$, using the moment technique introduced by Mathys. The application of the moment technique to the HARPS spectra allowed us in addition to study the presence of the crossover effect and quadratic magnetic fields. Our search for longitudinal magnetic fields resulted in first detections of weak magnetic fields in the Herbig Ae/Be stars HD 58647 and HD 98922. Further, we confirm the previous tentative detection of a weak magnetic field in HD 104237 by Donati et al. and confirm the previous detection of a magnetic field in the Herbig Ae star HD 190073. Surprisingly, the measured longitudinal magnetic field of HD 190073, $\left\langle B_{\mathrm{z}}\right\rangle=91 \pm 18 \mathrm{G}$ at a significance level of $5 \sigma$ is not in agreement with the measurement results of Alecian et al. (2013), $\left\langle B_{\mathrm{z}}\right\rangle=-10 \pm 20 \mathrm{G}$, who applied the LSD method to exactly the same data. No crossover effect was detected for any star in the sample. Only for HD 98922 the crossover effect was found to be close to $3 \sigma$ with a measured value of $-4228 \pm 1443 \mathrm{~km} \mathrm{~s}^{-1} \mathrm{G}$. A quadratic magnetic field of the order of $10 \mathrm{kG}$ was detected in HD 98922, and of $~ 3.5 \mathrm{kG}$ in HD 104237.
\end{abstract}

(C) 2013 WILEY-VCH Verlag GmbH \& Co. KGaA, Weinheim

\section{Introduction}

Herbig Ae/Be stars are pre-main-sequence objects with pronounced emission line features and an infrared excess indicative of dust in the circumstellar disks. Despite the general notion that they do not have convectively stable envelopes to support classical T Tauri-star-like dynamo action, in the last years it was demonstrated that a number of Herbig $\mathrm{Ae} / \mathrm{Be}$ stars have globally organised rather strong magnetic fields (e.g., Hubrig et al.2004; Wade et al.2005,2007; Alecian et al. 2009, Hubrig et al. 2009,2011a).

The evidence of large-scale organised magnetic fields detected in Herbig Ae/Be stars is reminiscent of the classical Ap/Bp stars, of which the Herbig Ae/Be stars may be precursors. Magnetic fields in these stars might be fossil of the early star formation epoch in which the magnetic field of the parental magnetised core was compressed into the innermost regions of the accretion disks (e.g. Banerjee \& Pudritz 2006). Alternatively, Tout \& Pringle (1995) proposed a nonsolar dynamo that could operate in rapidly rotating A-type stars based on rotational shear energy.

In our recent work based on low-resolution polarimetric spectra $(R=2000)$ obtained with the multi-mode instrument FORS 1 at the VLT (Hubrig et al. 2009), we found a

\footnotetext{
* Based on data obtained from the ESO Science Archive Facility under requests MSCHOELLER 51301, 51324, 36608-36611.

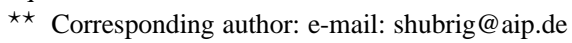

hint that stronger magnetic fields appear in very young Herbig $\mathrm{Ae} / \mathrm{Be}$ stars, and that the magnetic fields become very weak at the end of their PMS life, confirming the conclusions of Hubrig et al. (2000, 2005, 2007) that magnetic fields in stars with masses less than $3 M_{\odot}$ are rarely found close to the ZAMS and that $\mathrm{kG}$ magnetic fields appear in A stars already evolved from the ZAMS.

A series of mean longitudinal magnetic-field measurements for the Herbig Ae/Be stars HD 97048, HD 150193 , and HD 176386 obtained at low resolution with FORS $2 \sqrt{1}$ have recently been presented by Hubrig et al. (2011a). This study indicated that dipole models provide a satisfactory fit to the acquired magnetic data. On the other hand, the work of Adams \& Gregory (2012) shows that high order field components may even play a dominant role in the physics of the gas inflow, as the accretion columns approach the star.

Magnetically controlled behaviour has been found in both the spectroscopic and photometric variability of the strongly magnetic Herbig Ae star HD 101412 (Hubrig et al. 2010, 2011b). The small amount of UVES spectra acquired for this star have been analysed uncovering variations in equivalent widths, radial velocities, line widths, line asymmetries, and mean magnetic field modulus over the rotation period of $42.1 \mathrm{~d}$. Clearly, knowledge of the magnetic field structure combined with the determination of chemi-

\footnotetext{
${ }^{1}$ The spectropolarimetric capabilities of FORS 1 were moved to FORS 2 in 2009.
} 
cal composition (e.g., Hubrig et al. 2010, 2012, Cowley et al. 2010, Folsom et al. 2012) are indispensable to constrain theories on star formation and magnetospheric accretion in intermediate-mass stars.

Among the Herbig Ae/Be stars studied at low resolution with FORS 1 and reported as magnetic in previous studies, three Herbig Ae/Be stars, HD 97048, HD 100546, and HD 190073, have been observed during the last years at single epochs at very high spectral resolution $(R=$ $115,000)$ with the HARPS spectropolarimeter. They became now publically available in the ESO archive. Additionally, for three more Herbig Ae/Be stars, HD 58647, HD 98922, and HD 104237, single-epoch high-resolution HARPS spectropolarimetric observations have been acquired during the last years.

In this study we present our analysis of magnetic fields in this sample of six Herbig Ae/Be stars with spectral types between B9 and A4 and compare the measurements with those obtained from FORS 1/2 observations. For the analysis we use the moment technique developed by Mathys (e.g., Mathys 1991, 1995a, 1995b), which allows us not only the determination of the mean longitudinal magnetic field, but also to prove the presence of crossover effect and quadratic magnetic fields. Conveniently, among the sample stars, one star, HD 190073, has been reported as magnetic using the so-called Least-Squares Deconvolution (LSD) technique by Catala et al. (2007). Another star, HD 104237, was studied with the same technique by Donati et al. (1997), who achieved a marginal magnetic detection. These stars are used as a consistency check of our measurements.

\section{Reduction of HARPS spectra and notes on the sample}

The data for all six Herbig Ae/Be stars have been obtained in the polarimetric configuration of HARPS (Snik et al. 2011), yielding a spectral resolution of 115,000 . The targets for which we present spectropolarimetric measurements, together with their visual magnitudes, dates of observations, projected rotational velocities, and the achieved signal-tonoise ratios (SNR) are presented in Table 1. The HARPS archive spectra cover the wavelength range 3780-6913 $\AA$, with a small gap around $5300 \AA$. All spectra were recorded as sequences of individual subexposures taken at four different orientations of the quarter-wave retarder plate relative to the beam splitter of the polarimeter. The reduction was performed using the HARPS data reduction software available at the ESO headquarters in Germany.

To normalise the HARPS spectra to the continuum level, we used the image of the extracted echelle orders packed in one FITS file. First, we fit a continuum spline in columns of the image in cross-dispersion direction. Each column is fitted in a number of subsequent iterations until it converges to the same upper envelope of the continuum level. After each iteration, we analyze the residuals of the fit and make a robust estimation of the noise level based upon a statistical test of the symmetric part of the distribution. All pixels whose residuals are below the specified sigma clipping level are masked out from the subsequent fit. This way the smooth spline function is rejecting all spectral lines below, but leaving the continuum pixels to fit. Once all columns are processed, we fit the resulting smoothed curves in the dispersion direction by using the same approach with the robust noise estimation from the residuals, but this time rejecting possible outliers above and below the specified sigma clipping level. As a result, we create a bound surface with continuous first derivatives in the columns and rows. We employ a smoothing spline with adaptive optimal regularisation parameters, which selects the minimum of the curvature integral of the smoothing spline. As a test for the validity of the continuum fit, we check whether the normalised overlapping echelle orders are in good agreement with each other. The same is applied to the very broad hydrogen lines, whose winds may span over two or even three spectral orders. The typical mismatch between the red and blue ends of the neighboring orders is well within the statistical noise of these orders. The usual procedure to normalise a series of polarimetric observations of the same target but with different angles of the retarder, is to create a sum of the individual observations, normalise it to the continuum in the way described above, and to use the master normalised image as a template for the individual observations: by taking the ratio and fitting a regular spline to it, which then finally defines the continuum surface for the individual observation.

HD 58647 is probably the youngest Herbig Ae/Be star in our sample. Mendigutía et al. (2012) assign to this star $T_{\text {eff }}=10,500 \mathrm{~K}$ and an age of $0.4 \mathrm{Myr}$. According to the classification scheme of Meeus et al. (2001), this star with a self-shadowed disk belongs to group II. Mendigutía et al. (2011) did not detect any spectroscopic variability of $\mathrm{H} \alpha$, [O I] 6300, He I 5876 and Na I D lines on three consecutive nights, but noted in their work that variability on timescales longer than three days cannot be excluded. The profile of the $\mathrm{H} \alpha$ line from the HARPS spectrum presented on the left side in Fig. 1 exhibits a somewhat different shape compared to that displayed in the work of Mendigutía et al. in Fig. B.1. The profile is double-peaked with a much deeper sharp central absorption component. Such a deep sharp central absorption component is also evident in the $\mathrm{H} \beta$ profile displayed on the right side of Fig. 1 Clearly, additional highresolution spectroscopic observations are needed to solve the issue of variability.

Linear spectropolarimetric data of Mottram et al. (2007) reveal that the $\mathrm{H} \alpha$ polarisation behaviour is very different in Herbig Be stars from that of Herbig Ae or T Tauri stars. Only the Herbig Be star HD 58647 displays a line-effect at $\mathrm{H} \alpha$ and shows a loop in the QU diagram, similar to Herbig Ae stars within a magnetospheric accretion scenario. On the other hand, there are hints in the literature that HD 58647 is significantly older and could be a Vega-like star (Malfait et al. 1998) or a classical Be star (Manoj et al. 2002). The HARPS spectrum shows a fairly rapidly rotating star 
Table 1 The list of the Herbig Ae/Be stars observed with HARPSpol during the last years. The spectral types for five targets are taken from SIMBAD. For HD 104237, the spectral type was taken from Mora et al. (2001).

\begin{tabular}{rccccrc}
\hline \hline $\begin{array}{c}\text { HD } \\
\text { number }\end{array}$ & $\begin{array}{c}\text { Other } \\
\text { Identifier }\end{array}$ & $\begin{array}{c}\text { Spectral } \\
\text { type }\end{array}$ & $m_{V}$ & MJD & $\begin{array}{c}v \sin i \\
{\left[\mathrm{~km} \mathrm{~s}^{-1}\right]}\end{array}$ & SNR \\
\hline 58647 & BD-132008 & B9IV & 6.85 & 55906.2225 & $118^{1}$ & 437 \\
97048 & CD-76488 & A0 & 8.46 & 55707.9944 & $140^{2}$ & 177 \\
98922 & CD-524340 & B9V & 6.76 & 55706.0129 & $50^{3}$ & 379 \\
100546 & CD-69 893 & B9V & 6.70 & 55707.0335 & $65^{4}$ & 342 \\
104237 & DX Cha & A4 & 6.59 & 55319.2141 & $8^{5}$ & 170 \\
190073 & BD 05 4393 & A2IV & 7.73 & 55705.4291 & $0-8.6^{6}$ & 162 \\
\hline
\end{tabular}

Notes:

Sources for the $v \sin i$-values are: ${ }^{1}$ Mora et al. (2001), ${ }^{2}$ Böhm \& Catala (1995), ${ }^{3}$ Alecian et al. (2013a), ${ }^{4}$ Donati et al., (1997),

${ }^{5}$ Cowley et al. (2013), ${ }^{6}$ Catala et al. (2007).
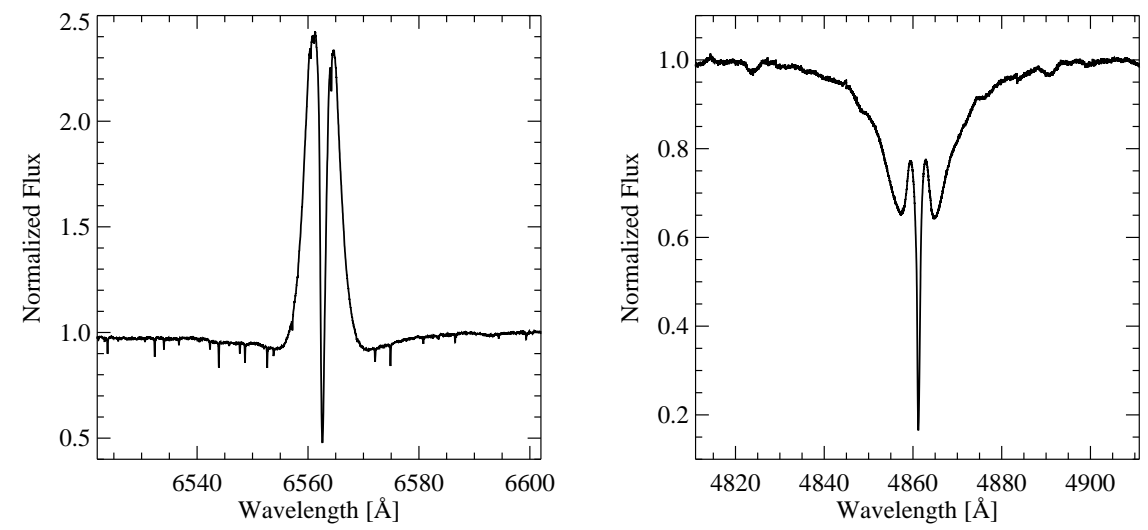

Fig. 1 Line profiles of $\mathrm{H} \alpha$ (left) and $\mathrm{H} \beta$ (right) with deep sharp central absorption components in the HARPS spectrum of the Herbig Ae/Be star HD 58647.
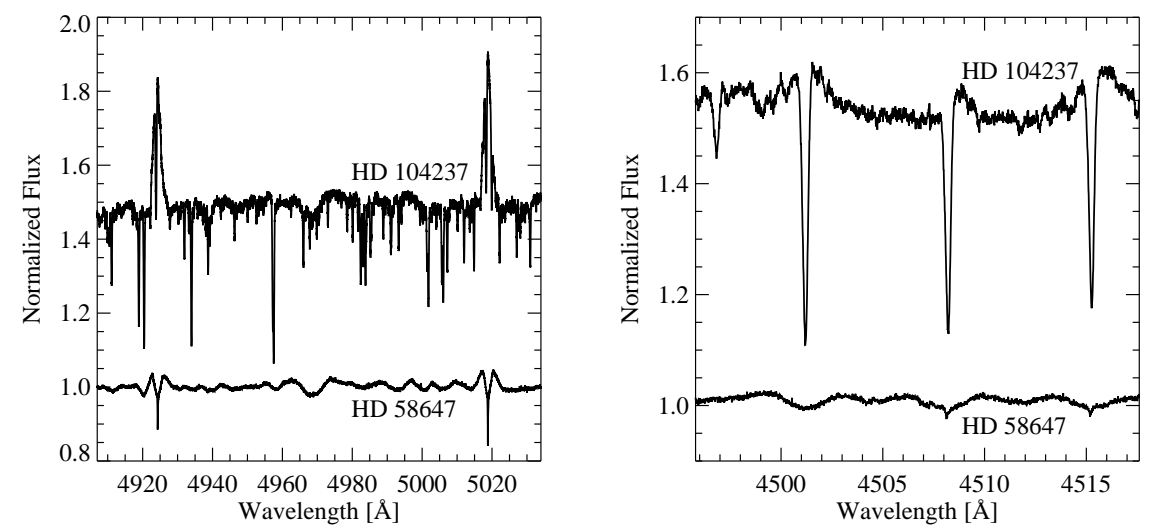

Fig. 2 Sharp absorption components in the HARPS spectrum of HD 58647 (lower spectrum). Left: Strong absorption components in the cores of the CS sensitive Fe II multiplet 42 lines at $4923 \AA$ and $5018 \AA$. Right: Less deep features in the cores of the Fe II lines at $4508 \AA$ (multiplet 38) and $4515 \AA$ (multiplet 37). Note the complete absence of such a feature in the Ti II multiplet 31 line at $4501 \AA$. To simplify the identification of the sharp features in the cores of the iron lines, we display in the upper spectra the same spectral region in the sharp-lined Herbig Ae star HD 104237. Please note that the upper spectrum is shifted in vertical direction for clarity. 


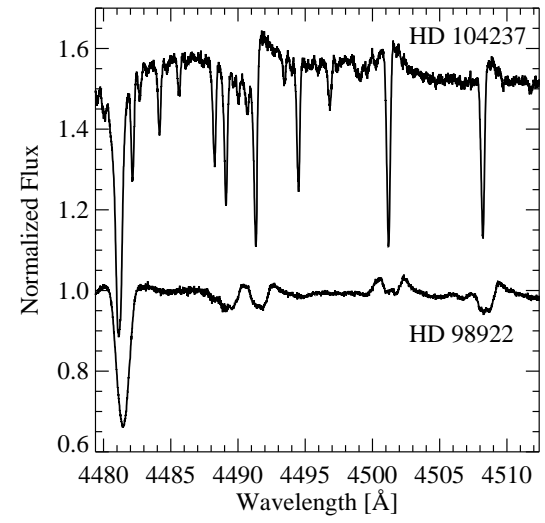

Fig. 4 Traces of small splitting in a few Fe II lines and the Ti II $\lambda 4501$ line in the HARPS spectrum of HD 98922 (lower spectrum). No splitting is observed in the Mg II $\lambda 4481$ line. To simplify the identification of the spectral lines, we present in the upper part the HARPS spectrum of the sharp-lined Herbig Ae star HD 104237 in the same wavelength region. Please note that the upper spectrum is shifted in vertical direction for clarity.

with strong, sharp and deep absorption components of Ca II $\mathrm{H}$ and $\mathrm{K}$ centered within the normal, broadened $\mathrm{H}$ and $\mathrm{K}$ lines, very similar to those detected in the star $\beta$ Pictoris (e.g. Slettebak 1975). A similar feature is visible in the $\mathrm{CH}(+) 4232 \AA$ line and in the Na I D lines. Sharp, but less deep absorption components are also detected in numerous Fe II lines. A few examples are presented in Fig. 2] Whether HD 58647 is a typical young Herbig Be star or belongs to the group of $\beta$ Pictoris stars needs further investigation.

HD 97048 is the fastest rotating star in our sample with $v \sin i=140 \mathrm{~km} \mathrm{~s}^{-1}$. This star illuminates the reflection nebula Ced 111 and is the brightest member of a cluster of young stars and the center of ongoing low-mass star formation (Habart et al. 2003). The disk properties of HD 97048 have been intensively studied in the recent years. Acke \& van den Ancker (2006) resolved extended [O I] line emission consistent with a disk with a semi-major axis oriented at a position angle of $160^{\circ}$. Similar to their work, also in the HARPS spectrum the [O I] line emission profiles are double-peaked. The modeled line profile in the work of Acke \& van den Ancker (2006) indicates that the doublepeaked lines are caused by the rotation of the disk. In Fig. 3 we present the normalised HARPS spectra of HD 97048 and another Herbig Ae/Be star in our sample, HD 98922, at the position of forbidden [O I] emission line profiles. HD 98922 is the only other Herbig Ae/Be star in our sample showing double-peaked [O I] emission lines.

In the HARPS spectrum of HD 98922 an interesting fact is that the Fe II and the Ti II lines show traces of splitting, best visible in the Ti II lines. Lines belonging to different elements exhibit different shapes of their profiles. Such a behaviour suggests that it is possible that some elements are inhomogeneously distributed on the stellar surface. A similar behaviour of line profiles was detected in the spectra of the strongly magnetic Herbig Ae star HD 101412 (Hubrig et al. 2010), for which the most pronounced variability was detected for spectral lines of He I and the iron peak elements, whereas the spectral lines of CNO elements were only slightly variable. In Fig. 4 4 we present the line profiles of Mg II $\lambda 4481$, a few Fe II lines, and the Ti II $\lambda 4501$ line. The interferometric study of the $\mathrm{Br} \gamma$ line-emitting region in HD 98922 by Kraus et al. (2008) revealed that this region is compact enough to support the hypothesis that most of the $\mathrm{Br} \gamma$-emission emerges from magnetospheric accretion columns.

Based on the age estimate of van den Ancker et al. (1998, >10 Myr), HD 100546 is one of the oldest targets in the sample. Near-infrared coronagraphic observations of HD 100546 detected a disk extending to $515 \mathrm{AU}$ (Grady et al.2001) with an inclination of $49^{\circ}$. According to Bouwman et al. (2003), the spectral appearance and dust properties of HD 100546 differ from those of other Herbig Ae/Be stars, showing a large fraction of forsterite and an almost identical grain composition as the comet Hale-Bopp. Modeling of the SED (Bouwman et al.2003) and imagery with the Hubble Space Telescope (Grady et al. 2005) indicate the presence of an inner hole in the dust distribution at 10-13 AU. Also the study of the spectral profile of the [O I] $\lambda 6300$ line by Acke \& van den Ancker (2006) suggested that a gap is present at $10 \mathrm{AU}$ in the disk around this star, and such a gap is likely planet-induced. Recent high-contrast observations of the circumstellar environment of HD 100546 by Quanz et al. (2013) are interpreted by the presence of a planet in the process of forming.

The Herbig Ae/Be star HD 104237 was intensively studied during the last years using multi wavelength observations, in particular due to the possible presence of a magnetic field announced more than 15 years ago by Donati et al. (1997). In their work the authors reported on the probable detection of a weak magnetic field of the order of $50 \mathrm{G}$. Imaging observations by Grady et al. (2004) suggested that the disk of HD 104237 is seen nearly face-on. The star is a primary in an SB2 system with an orbital period of $19.86 \mathrm{~d}$ (Böhm et al.2004). A recent study of chemical abundances in both components is presented by Cowley et al. (2013).

The absorption and emission spectrum of the Herbig Ae/Be star HD 190073 was studied in detail by Catala et al. (2007) and more recently by Cowley \& Hubrig (2012). The low projected rotational velocity $\left(v \sin i \leq 9 \mathrm{~km} \mathrm{~s}^{-1}\right)$ may indicate either a very slow rotation, or a very small inclination of the rotation axis with respect to the line of sight. Recent interferometric observations in the infrared are best interpreted in terms of a circumstellar disk seen nearly faceon (Eisner et al.2004). Also IUE observations indicate a low inclination angle of the rotation axis (Hubrig et al.2009).

Baines et al. (2006) applied the technique of spectroastrometry to study the presence of close companions in a sample of Herbig Ae/Be stars, which also includes all six 

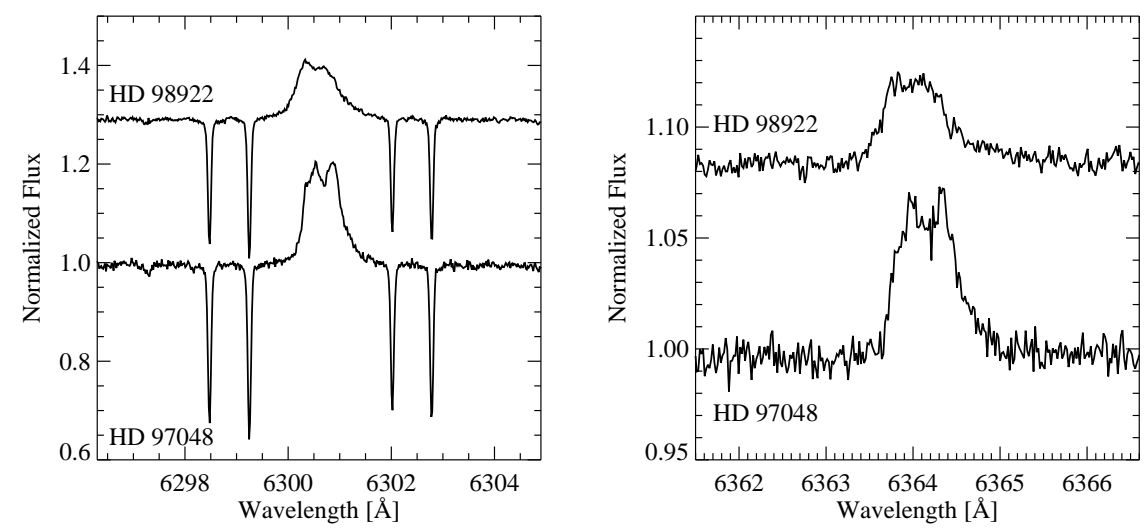

Fig. 3 Double-peaked forbidden [O I] emission line profiles at $6300 \AA$ and $6363 \AA$ in the HARPS spectra of the Herbig Ae/Be stars HD 97048 (lower spectra) and HD 98922 (upper spectra). Please note that the upper spectra are shifted in vertical direction for clarity.

Herbig Ae/Be stars studied in this work. The method is particularly suited to detect binary companions of emissionline stars and can detect companions as much as 6-mag fainter than the primary. The presence of a companion was detected for HD 58647 and HD 98922, while possible binary detections have been mentioned for HD 104237 and HD 190073. According to the work of Böhm et al. (2004) and Cowley et al. (2013), HD 104237 is a SB2 system where lines of the secondary are clearly identified in the spectrum. No close companions have been reported for HD 97048 and HD 100546.

\section{Magnetic field measurements}

The Stokes $I$ and $V$ parameters were derived following the ratio method described by Donati et al. (1997), ensuring in particular that all spurious signatures are removed at first order. Null polarisation spectra (labeled with null in Table 3) have been calculated by combining the sub-exposures in such a way that the polarisation cancels out, allowing us to verify that no spurious signals are present in the data.

Wavelength shifts between right- and left-hand side circularly polarised spectra were interpreted in terms of a longitudinal magnetic field $\left\langle B_{\mathrm{z}}\right\rangle$, using the moment technique described by Mathys (1994). All the lines we tried to employ in the diagnosis of the magnetic fields on the surface of our target stars are presented in Table 2, together with their Landé factors. The wavelengths and the Landé factors were taken from Kurucz's list of atomic data (Kurucz 1989). We note that the actual list of lines measured in the spectrum of each star can differ from one observation to the next due to the different quality of the spectra, contamination by the CS material, and the projected rotational velocity where blend-free spectral lines are difficult to identify in fast rotating targets. All stars have very different line widths, with the sharpest lines in HD 190073 and HD 104237. The other four stars have $v \sin i$ values $\geq 50 \mathrm{~km} \mathrm{~s}^{-1}$. The contami-

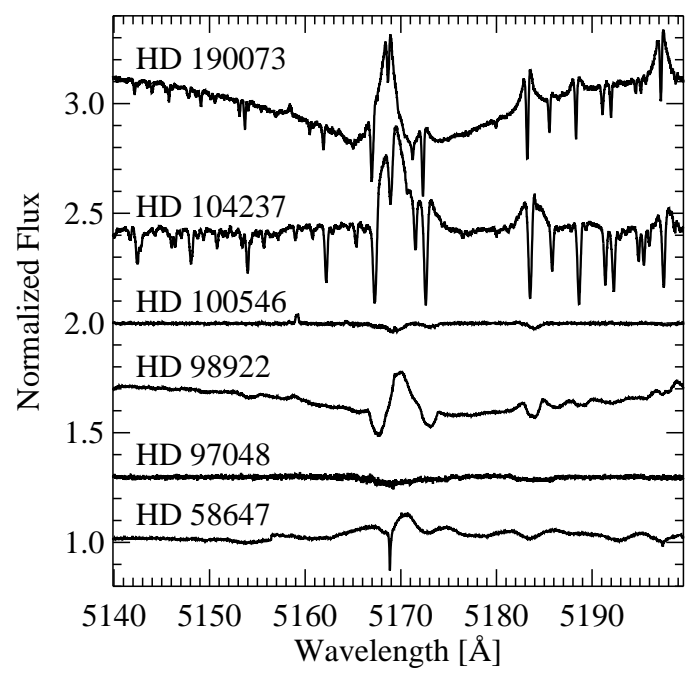

Fig. 5 The behaviour of the Fe II multiplet 42 line at $\lambda 5169$ in all studied stars. From bottom to the top we present the HARPS spectra of HD 58647, HD 97048, HD 98922, HD 100546, HD 104237, and HD 190073. Please note that the spectra are shifted in vertical direction for clarity.

nation by the circumstellar (CS) material is different in different stars. The CS environment of the Herbig Ae/Be stars typically consist of a combination of disk, wind, accretion, and jets. As an example of the different impact of the CS material on the line profiles in our sample stars, we present in Fig. 5 the spectral region with the profiles of the Fe II $\lambda 5169$ lines of multiplet 42 .

The measurements of the mean longitudinal magnetic field $\left\langle B_{\mathrm{z}}\right\rangle$ together with the mean longitudinal magnetic field determined from null spectra $\left\langle B_{\mathrm{z}}\right\rangle_{\text {null }}$ using HARPS spectropolarimetric observations are listed in Table 3 . The 
Table 2 Line list for the different elements used in the magnetic field measurements.

\begin{tabular}{|c|c|c|c|c|c|c|c|c|}
\hline $\begin{array}{c}\text { Wavelength } \\
[\AA]]\end{array}$ & $\begin{array}{l}\text { Landé } \\
\text { Factor }\end{array}$ & Element & $\begin{array}{c}\text { Wavelength } \\
{[\AA]}\end{array}$ & $\begin{array}{l}\text { Landé } \\
\text { Factor }\end{array}$ & $\overline{\text { Element }}$ & $\begin{array}{c}\text { Wavelength } \\
{[\AA]}\end{array}$ & $\begin{array}{l}\text { Landé } \\
\text { Factor }\end{array}$ & Element \\
\hline 4002.5430 & 1.218 & Fe II & 4294.0991 & 1.203 & Ti II & 4571.9678 & 0.944 & Ti II \\
\hline 4005.2410 & 1.484 & $\mathrm{Fe} \mathrm{I}$ & 4307.9019 & 1.121 & $\mathrm{Fe} I$ & 4576.3398 & 1.184 & Fe II \\
\hline 4012.3850 & 0.716 & Ti II & 4383.5439 & 1.149 & Fe I & 4588.1992 & 1.059 & Cr II \\
\hline 4057.4609 & 0.890 & Fe II & 4385.3872 & 1.330 & Fe II & 4592.0488 & 1.201 & Cr II \\
\hline 4122.6680 & 1.326 & Fe II & 4399.7720 & 1.400 & Ti II & 4616.6289 & 0.793 & CrII \\
\hline 4132.0581 & 1.510 & $\mathrm{Fe} \mathrm{I}$ & 4404.7500 & 1.129 & $\mathrm{Fe} I$ & 4618.8032 & 0.914 & Cr II \\
\hline 4134.6758 & 1.194 & $\mathrm{Fe} \mathrm{I}$ & 4415.1221 & 1.104 & $\mathrm{Fe} I$ & 4620.5210 & 1.305 & Fe II \\
\hline 4143.4150 & 1.032 & $\mathrm{Fe} I$ & 4416.8301 & 0.767 & Fe II & 4629.3389 & 1.314 & Fe II \\
\hline 4145.7808 & 1.173 & Cr II & 4464.4502 & 0.493 & Ti II & 4634.0698 & 0.508 & Cr II \\
\hline 4175.6362 & 1.145 & $\mathrm{Fe} I$ & 4489.1831 & 1.386 & Fe II & 4635.3159 & 1.042 & Fe II \\
\hline 4202.0288 & 1.175 & $\mathrm{Fe} I$ & 4491.4048 & 0.421 & Fe II & 4666.7578 & 1.513 & Fe II \\
\hline 4242.3638 & 1.200 & Cr II & 4508.2881 & 0.503 & Fe II & 4731.4531 & 0.655 & Fe II \\
\hline 4250.1182 & 1.502 & $\mathrm{Fe} I$ & 4515.3389 & 1.044 & Fe II & 4755.7271 & 1.058 & Mn II \\
\hline 4260.4731 & 1.591 & $\mathrm{Fe} \mathrm{I}$ & 4520.2241 & 1.337 & Fe II & 4779.9849 & 1.375 & Ti II \\
\hline 4271.7588 & 1.236 & Fe I & 4522.6338 & 0.921 & Fe II & 4812.3369 & 1.499 & CrII \\
\hline 4275.5669 & 0.922 & Cr II & 4541.5239 & 0.774 & Fe II & 4824.1270 & 1.339 & Cr II \\
\hline 4282.4019 & 1.347 & $\mathrm{Fe} \mathrm{I}$ & 4558.6499 & 1.161 & Cr II & 4923.9268 & 1.695 & Fe II \\
\hline 4284.1880 & 0.521 & CrII & 4563.7612 & 0.985 & Ti II & 5018.4399 & 1.933 & Fe II \\
\hline
\end{tabular}

Table 3 Measurements of the mean longitudinal magnetic field in our sample stars. All quoted errors are $1 \sigma$ uncertainties.

\begin{tabular}{lrr}
\hline \hline Object & \multicolumn{1}{c}{$\left\langle B_{\mathrm{z}}\right\rangle$} & \multicolumn{1}{c}{$\left\langle B_{\mathrm{z}}\right\rangle_{\text {null }}$} \\
& \multicolumn{1}{c}{$[\mathrm{G}]$} & \multicolumn{1}{c}{$[\mathrm{G}]$} \\
\hline HD 58647 & $218 \pm 69$ & $54 \pm 71$ \\
HD 98922 & $-131 \pm 34$ & $-42 \pm 36$ \\
HD 104237 & $63 \pm 15$ & $-5 \pm 17$ \\
HD 190073 & $91 \pm 18$ & $21 \pm 18$ \\
\hline
\end{tabular}

presence of a weak positive longitudinal magnetic field $\left\langle B_{\mathrm{z}}\right\rangle=218 \pm 69 \mathrm{G}$ at a significance level of $3.2 \sigma$ in HD 58647 is reported here for the first time. Due to the presence of sharp narrow components in a number of spectral lines, only the lines without visually noticeable contribution of circumstellar material have been selected for magnetic field measurements.

We were not able to determine a longitudinal magnetic field in the HARPS spectra of the Herbig Ae/Be stars HD 97048 and HD 100546. The HARPS spectrum of HD 97048 retrieved from the ESO archive was obtained at a rather low SNR. Since HD 97048 is the fastest rotating target in our sample, a high SNR, up to a few hundreds, is indispensable to detect a magnetic field. As for HD 100546, we experienced the same surprise as Donati et al. (1997) finding only very few photospheric features in the spectrum, but a number of forbidden emission [Fe II] lines. In addition to the forbidden emission [Fe II] lines belonging to multiplets $19 \mathrm{~F}$ and $20 \mathrm{~F}$ identified by Donati et al. (1997), in our HARPS spectrum we have detected also forbidden emission [Fe II] lines belonging to multiplets $6 \mathrm{~F}$ and 7F. Some of the detected emission lines appear very symmetric, although there is a number of line profiles of [Fe II] in the

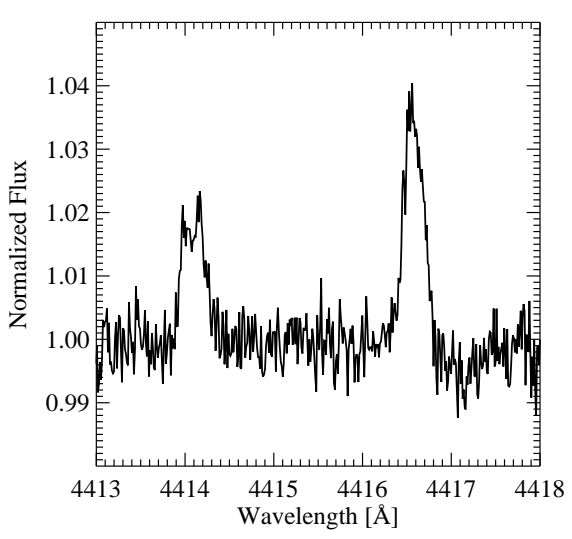

Fig. 6 Forbidden [Fe II] emission lines of multiplets $6 \mathrm{~F}$ and $7 F$ in the wavelength region $4413-4417 \AA$ in the HARPS spectrum of HD 100546. Note that the doublepeaked profile at $\lambda 4414$ consists of two emission lines, $\lambda 4413.79$ (multiplet 7F) and $\lambda 4414.45$ (multiplet 6F).

HARPS spectrum showing asymmetric line profiles. Also the forbidden emission [O I] lines show slightly asymmetric profiles. In Fig. 6 we present the spectral region around the forbidden emission [Fe II] lines belonging to multiplets $6 \mathrm{~F}$ and $7 \mathrm{~F}$. An example of an asymmetric profile of the forbidden emission [O I] line at $\lambda 6393$ is displayed in Fig. 7 No magnetic field was detected in HD 100546 by Donati et al. (1997) using the LSD technique. Our magnetic field measurements using just a few photospheric lines cannot be considered reliable due to the large measurement inaccuracy and are not presented in Table 3 .

On the other hand, Hubrig et al. (2009,2011a) reported the detection of weak longitudinal magnetic fields in both 


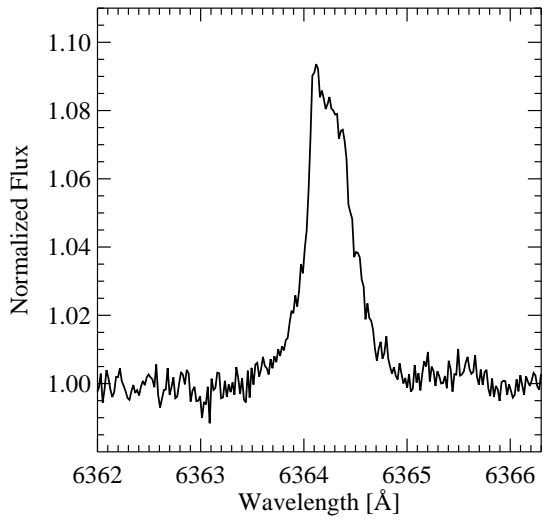

Fig. 7 Asymmetric line profile of the forbidden [O I] emission line at $6363 \AA$ in the HARPS spectrum of HD 100546.

Herbig Ae/Be stars HD 97048 and HD 100546 using lowresolution spectra obtained in spectropolarimetric mode with FORS $1 / 2$ mounted on the VLT. Using multi-epoch polarimetric spectra for HD 97048, the authors detected a variation of the longitudinal magnetic field in the range from $-186 \mathrm{G}$ to $164 \mathrm{G}$ and suggest a magnetic/rotation period of $0.693 \mathrm{~d}$. The longitudinal magnetic field of HD 100546 appears to be much weaker, only of the order of $90 \mathrm{G}$. Clearly, additional high-quality spectropolarimetric observations are needed to confirm or disprove the presence of a magnetic field in these stars.

Also for the first time we present here the detection of a longitudinal magnetic field $\left\langle B_{\mathrm{z}}\right\rangle=-131 \pm 34 \mathrm{G}$ at a significance level of $3.9 \sigma$ in the moderately rotating Herbig Ae/Be star HD 98922. This star was previously observed only once by Wade et al. (2007) who have not detected the presence of a magnetic field using low-resolution FORS 1 spectra.

The best accuracy in the magnetic field determination using HARPS spectra is achieved in both sharp-lined stars HD 104237 and HD 190073. Donati et al. (1997) detected a marginal Zeeman signature in the LSD Stokes $V$ profile of HD 104237 in the spectra obtained with a visitor polarimeter coupled to the UCL Echelle Spectrograph at AAT. Our measurement, $\left\langle B_{\mathrm{z}}\right\rangle=63 \pm 15 \mathrm{G}$, is the first confirmation of the presence of a magnetic field in this star.

The first measurement of a longitudinal magnetic field in HD 190073 was published by Hubrig et al. (2006) indicating the presence of a longitudinal magnetic field $\left\langle B_{\mathrm{z}}\right\rangle=$ $84 \pm 30 \mathrm{G}$ measured on FORS 1 low-resolution spectra at $2.8 \sigma$ level. Later on this star was studied by Catala et al. (2007) using ESPaDOnS observations, who confirmed the presence of a weak magnetic field, $\left\langle B_{\mathrm{z}}\right\rangle=74 \pm 10 \mathrm{G}$, at a higher significance level. A few years later a longitudinal magnetic field $\left\langle B_{\mathrm{z}}\right\rangle=104 \pm 19 \mathrm{G}$ was reported by Hubrig et al. (2009) using FORS 1 measurements. The measurement of the longitudinal magnetic field using the available archival HARPS observations from May 2011, $\left\langle B_{\mathrm{z}}\right\rangle=91 \pm 18 \mathrm{G}$ presented in this work fully confirms the presence of a rather stable weak field.

Surprisingly, new observations of this star during July 2011 and October 2012 by Alecian et al. (2013b) detected variations of the Zeeman signature in the LSD spectra on timescales of days to weeks. Among the Zeeman signatures displayed in their Fig. 1, the LSD Zeeman feature extracted from the same HARPS spectrum as in our analysis is also presented, but the strength of the measured longitudinal magnetic field is only $\left\langle B_{\mathrm{z}}\right\rangle=-10 \pm 20 \mathrm{G}$. The authors suggest that the detected variations of Zeeman signatures are the result of the interaction between the fossil field and the ignition of a dynamo field generated in the newly-born convective core. As our measurements completely contradict those presented by Alecian et al., careful spectropolarimetric monitoring over the next years is important to confirm the reported variability of the magnetic field.

No crossover effect was detected for any star in the sample. The crossover effect is measured by the second-order moment about the centre of the profiles of spectral lines recorded in the Stokes parameter $V$ (Mathys 1995a). Only for HD 98922 the crossover effect was found to be close to $3 \sigma$ with a measured value of $-4228 \pm 1443 \mathrm{~km} \mathrm{~s}^{-1} \mathrm{G}$.

A quadratic magnetic field of the order of $10 \mathrm{kG}$ $\left(\left\langle B_{\mathrm{q}}\right\rangle=10,246 \pm 3246 \mathrm{G}\right)$ was detected in HD 98922, and of $\sim 3.6 \mathrm{kG}\left(\left\langle B_{\mathrm{q}}\right\rangle=3562 \pm 1164 \mathrm{G}\right)$ in HD 104237 . A quadratic magnetic field close to the $3 \sigma$ level, $\left\langle B_{\mathrm{q}}\right\rangle=$ $3169 \pm 1132 \mathrm{G}$ was measured for HD 190073. The mean quadratic magnetic field,

$$
\left\langle B_{q}\right\rangle=\left(\left\langle B^{2}\right\rangle+\left\langle B_{z}^{2}\right\rangle\right)^{1 / 2},
$$

is derived through the application of the moment technique, as described e.g. by Mathys \& Hubrig (1995). Here, $\left\langle B^{2}\right\rangle$ is the mean square magnetic field modulus, i.e. the average over the stellar disc of the square of the modulus of the magnetic field vector, weighted by the local emergent line intensity, while $\left\langle B_{z}^{2}\right\rangle$ is the mean square longitudinal magnetic field, i.e. the average over the stellar disc of the square of the line-of-sight component of the magnetic vector, weighted by the local emergent line intensity. Importantly, contrary to the mean longitudinal magnetic field, the mean quadratic magnetic field provides a measurement of the field strength that is fairly insensitive to its structure.

\section{Discussion}

With this work we increase the number of Herbig Ae/Be stars showing the presence of magnetic fields. Longitudinal magnetic fields have been detected in about a dozen Herbig Ae/Be stars (e.g., Hubrig et al. 2004, 2006, 2007, 2009, 2011a, 2011b, Wade et al. 2007, Catala et al. 2007, Alecian et al.2009). For the majority of these stars rather small magnetic fields were measured, of the order of only $100 \mathrm{G}$ or less. Presently, the Herbig Ae star HD 101412 possesses the strongest longitudinal magnetic field ever measured in any Herbig Ae star, with a surface magnetic field $\langle B\rangle$ up to 
S. Hubrig et al.: HARPS spectropolarimetry of Herbig Ae/Be stars

$3.5 \mathrm{kG}$. HD 101412 is also the only Herbig Ae/Be star for which the rotational Doppler effect was found to be small in comparison to the magnetic splitting and several spectral lines observed in unpolarised light at high dispersion are resolved into magnetically split components (Hubrig et al. 2010, 2011b).

To understand the magnetospheres of Herbig Ae/Be stars and their interaction with the circumstellar environment presenting a combination of disk, wind, accretion, and jets, the knowledge of the magnetic field strength and topology is indispensable. Progress in understanding the diskmagnetosphere interaction can, however, only come from studying a sufficient number of targets in detail to look for patterns encompassing this type of pre-main sequence stars.

In the presented high-resolution HARPS spectra, the contamination of the spectra by the CS material is clearly visible, and it is of utmost importance to study the disk-magnetosphere interaction in detail by modeling the Stokes $V$ profiles not only in photospheric lines, but also in various wind and accretion diagnostic lines. The Dopplershifted spectropolarimetric contributions from photospheric and circumstellar environmental diagnostic lines should be investigated using high-resolution polarimetric observations over the stellar rotation period. These studies will enable the application of the technique of Doppler Zeeman tomography to determine the correspondence between the magnetic field structure and the radial density and temperature profiles and to construct for the first time a realistic model of magnetospheres of Herbig Ae/Be stars that takes into account the full complexity of the circumstellar environment, including the observed outflows and jets.

Acknowledgements. This research has made use of the SIMBAD database, operated at CDS, Strasbourg, France.

\section{References}

Acke, B., van den Ancker, M.E.: 2006, A\&A 449, 267

Adams, F.C., Gregory, S.G.: 2012, ApJ 744, 55

Alecian, E., Wade, G.A., Catala, C., et al.: 2009, MNRAS 400, 354

Alecian, E., Wade, G.A., Catala, C., et al.: 2013a, MNRAS 429, 1001

Alecian, E., Neiner, C., Mathis, S., et al.: 2013b, A\&A 549, L8

Baines, D., Oudmaijer, R.D., Porter, J.M., Pozzo, M.: 2006, MNRAS 367,737

Banerjee, R., Pudritz, R.E.: 2006, ApJ 641, 949

Boehm, T., Catala, C.: 1995, A\&A 301, 155

Böhm, T., Catala, C., Balona, L., Carter, B.: 2004, A\&A 427, 907

Bouwman, J., de Koter, A., Dominik, C., Waters, L.B.F.M.: 2003, A\&A 401, 577

Catala, C., Alecian, E., Donati, J.-F., et al.: 2007, A\&A 462, 293

Cowley, C.R., Hubrig, S., González, J.F., Savanov, I.: 2010, A\&A 523, A65

Cowley, C.R., Hubrig, S.: 2012, Astr. Nach. 333, 34

Cowley, C.R., Castelli, F., Hubrig, S.: 2013, MNRAS in press, also arXiv:1303.1850

Donati, J.-F., Semel, M., Carter, B.D., et al.: 1997, MNRAS 291, 658
Eisner, J.A., Lane, B.F., Hillenbrand, L.A., et al.: 2004, ApJ 613, 1049

Folsom, C.P., Bagnulo, S., Wade, G.A., et al.: 2012, MNRAS 422, 2072

Grady, C.A., Polomski, E.F., Henning, T., et al.: 2001, AJ 122, 3396

Grady, C.A., Woodgate, B., Torres, C.A.O., et al.: 2004, ApJ 608, 809

Grady, C.A., Woodgate, B., Heap, S.R., et al.: 2005, ApJ 620, 470

Habart, E., Testi, L., Natta, A., Vanzi, L.: 2003, A\&A 400, 575

Hubrig, S., North, P., Mathys, G.: 2000, ApJ 539, 352

Hubrig, S., Schöller, M., Yudin, R.V.: 2004, A\&A 428, L1

Hubrig, S., Schöller, M., North, P.: 2005, In: "Magnetic Fields in the Universe: From Laboratory and Stars to Primordial Structures", American Institute of Physics Conf. Ser., Vol. 784, Eds. E. M. de Gouveia dal Pino, G. Lugones, \& A. Lazarian, p. 145

Hubrig, S., Yudin, R.V., Schöller, M., Pogodin, M.A.: 2006, A\&A 446, 1089

Hubrig, S., Pogodin, M.A., Yudin, R.V., et al.: 2007, A\&A 463, 1039

Hubrig, S., Stelzer, B., Schöller, M., et al.: 2009, A\&A 502, 283

Hubrig, S., Schöller, M., Savanov, I., et al.: 2010, Astron. Nach. 331,361

Hubrig, S., Schöller, M., Ilyin, I., et al.: 2011a, A\&A 536, A45

Hubrig, S., Mikulasek, Z., González, J.F., et al.: 2011b, A\&A 525, L4

Hubrig, S., Castelli, F., González, J.F., et al.: 2012, A\&A 542, A31

Kraus, S., Hofmann, K.-H., Benisty, M., et al.: 2008, A\&A 489, 1157

Kurucz, R.L.: 1989, http://kurucz.cfa.harvard.edu/atoms

Malfait, K., Bogaert, E., Waelkens, C.: 1998, A\&A 331, 211

Manoj, P., Maheswar, G., Bhatt, H.C.: 2002, MNRAS 334, 419

Mathys, G.: 1991, A\&AS 89, 121

Mathys, G.: 1994, A\&AS 108, 547

Mathys, G.: 1995a, A\&A 293, 733

Mathys, G.: 1995b, A\&A 293, 746

Mathys, G., Hubrig, S.: 1995, A\&A 293, 810

Meeus, G., Waters, L.B.F.M., Bouwman, J., et al.: 2001, A\&A 365, 476

Mendigutía, I., Eiroa, C., Montesinos, B., et al.: 2011, A\&A 529, A34

Mendigutía, I., Mora, A., Montesinos, B., et al.: 2012, A\&A 543, A59

Mora, A., Merín, B., Solano, E., et al.: 2001, A\&A 378, 116

Mottram, J.C., Vink, J.S., Oudmaijer, R.D., Patel, M.: 2007, MNRAS 377, 1363

Quanz, S.P., Amara, A., Meyer, M.R., et al.: 2013, ApJL 766, L1

Slettebak, A.: 1975, ApJ 197, 137

Snik, F., Kochukhov, O., Piskunov, N., et al.: 2011, In: "Solar Polarization 6", Astr. Soc. of the Pacific Conf. Ser., Vol. 437, Eds. J. R. Kuhn, D. M. Harrington, H. Lin, S. V. Berdyugina, J. Trujillo-Bueno, S. L. Keil, T. Rimmele, p. 237

Tout, C.A., Pringle, J.E.: 1995, MNRAS 272, 528

van den Ancker, M.E., de Winter, D., Tjin A Djie, H.R.E.: 1998, A\&A 330, 145

Wade, G.A., Drouin, D., Bagnulo, S., et al.: 2005, A\&A 442, L31

Wade, G.A., Bagnulo, S., Drouin, D., et al.: 2007, MNRAS 376, 1145 